\title{
A Miniaturized Stepwise Injection Spectrophotometric Analyzer
}

\author{
Anastasiia Petrova, *† Andrey Bulatov,* Andrey Vishnikin,** Leonid Moskvin,** \\ Ryoichi ISHIMATSU, $* * *$ Koji NAKANO, $* * *$ and Toshihiko IMATO*** \\ *Department of Analytical Chemistry, Institute of Chemistry, Saint-Petersburg State University, \\ Saint-Petersburg, pr. Universitetskij 26, 19850, Russia \\ **Department of Analytical Chemistry, Institute of Chemistry, Oles Honchar Dnipropetrovsk National \\ University, Gagarina 72, Dnipropetrovsk 49010, Ukraine \\ ***Department of Applied Chemistry, Graduate School of Engineering, Kyushu University, 744 Motooka, \\ Nishi, Fukuoka 819-0395, Japan
}

\begin{abstract}
A novel micro-stepwise injection analyzer ( $\mu$ SWIA) has been developed for the automation and miniaturization of spectrophotometric analysis. The main unit of this device is a mixing chamber (MC) connected to the atmosphere. This part of the $\mu$ SWIA provides rapid and effective homogenization of the reaction mixture components and completion of the reaction by means of gas bubbling. The $\mu$ SWIA contained a rectangular labyrinth channel designed in way allowing one to eliminate bubbles by moving a solution from the MC to an optical channel. The light-emitting diode (LED) was used as a light emitter and the analytical signal was measured by a portable spectrophotometer. Fluid movement was attained via the use of a computer-controlled syringe pump. The $\mu$ SWIA was successfully used for the spectrophotometric determination of cysteine in biologically active supplements and fodder by using 18-molybdo-2-phosphate heteropoly anion (18-MPA) as the reagent.
\end{abstract}

Keywords Stepwise injection spectrophotometric analysis, automation, miniaturization, determination of cysteine

(Received December 26, 2014; Accepted March 14, 2015; Published June 10, 2015)

\section{Introduction}

An important and rapidly growing trend existing in modern analytical chemistry is the miniaturization of analytical systems. Lately, the miniaturization of analytical procedures based on flow analysis is being development intensively. ${ }^{1-4}$ Therefore, labor costs for the analysis have decreased, and the volume of the sample solutions and reagents as well as generated waste are substantially reduced. Consequently, the miniaturization of flow analytical systems assisted in fulfilling the principles declared by "Green Chemistry". 5

Applying modern technologies to the miniaturization of flow analysis is usually realized based on a microfluidic chip. Micropumps, micro-valves, heating micro-devices and various detectors are directly integrated into a microchip. Manz et al. have proposed the micro total analytical system ( $\mu$ TAS) concept, opening a new era in the field of analytical chemistry. ${ }^{2}$ The $\mu$ TAS makes it possible to carry out analyses in flow systems having $\mu \mathrm{L}$ and even sub- $\mu \mathrm{L}$ volumes, so as to achieve analysis times on the order of seconds.

Microfluidic chips based on flow injection analysis ( $\mu$ FIA) have been developed. ${ }^{6}$ The $\mu$ FIA chip is characterized by simplicity and high throughput. However, due to sample dispersion and poor mixing, especially for multireagent and slow reactions, the sensitivity of the method is decreased.

$\dagger$ To whom correspondence should be addressed.

E-mail: petrovastacy@gmail.com
Moreover, the $\mu$ FIA is not flexible, and generally does not allow one to perform different analytical procedures without changing the topology of the chip.

For the miniaturization of sequential injection analysis, Ruzicka invented the lab-on-valve system. ${ }^{7}$ It has now been recognized as a universal tool for the accommodation of a wide variety of micro-scale analytical processes. ${ }^{8}$

Later, Cerda proposed a chip-on-valve for the miniaturization of multisyringe flow-injection analysis. ${ }^{9,10}$ The analyzer included a special panel equipped by four syringes for one-off moving of the sample and reagent solutions. This panel was coupled with a polymethylmethacrylate (PMMA) plate that contains a serpentine reaction coil for mixing solutions' and an optical channel for absorbance measurements.

A concept of performing a flow analysis by the use of a compact disk (CD) type was proposed. A CD-type microchip based on centrifugal force was successfully developed. ${ }^{11,12}$ Reservoirs for the solutions and channels were an integral part of the CD. In this case it was not necessary to use extra pumps. Complete, simple, fast and effective mixing of the components of the reaction mixture can be realized by using several flow approaches, including flow-batch analysis (FBA), ${ }^{13}$ zone fluidic analysis $^{14}$ and stepwise injection analysis (SWIA). ${ }^{15-18}$ These methods include mixing the solutions in a mixing chamber to achieve physical and chemical equilibrium and high sensitivity. Previously, only for the miniaturization of $\mathrm{FBA}^{19}$ a micro-flowbatch device was proposed. This device included microchannels for the injection of solutions into the micro-mixing chamber where the solutions were mixed by means of a fishing 
line connected to a CD drive motor. The micro-mixing chamber was integrated with a light-emitting diode (LED) and a phototransistor for absorbance measurements.

In this work, a new strategy has been presented for the automation and miniaturization of spectrophotometric analysis based on stepwise injection analysis. A new micro-stepwise injection analyzer was developed and its performance demonstrated by the determination of cysteine in biologically active supplements and fodders by its reaction with 18-molybdo2-phosphate heteropoly anion (18-MPA). ${ }^{18}$

\section{Experimental}

\section{Chemicals}

A stock solution of $0.01 \mathrm{M}$ cysteine hydrochloride (Wako Pure Chemical Industries, Japan) was prepared daily by accurately dissolving a weighed amount in $0.01 \mathrm{M} \mathrm{HCl}$ and stored in a refrigerator. $0.10 \mathrm{M}$ acetic acid and $0.10 \mathrm{M}$ sodium acetate were used for preparing an acetate buffer solution with $\mathrm{pH}$ 5.0. The ammonium salt of the 18-MPA, $\left(\mathrm{NH}_{4}\right)_{6} \mathrm{P}_{2} \mathrm{Mo}_{18} \mathrm{O}_{62}$ $\times 14 \mathrm{H}_{2} \mathrm{O}$, was synthesized according to a described procedure. ${ }^{15}$ A $0.01 \mathrm{M}$ solution of 18-MPA was prepared by dissolving $0.7855 \mathrm{~g}$ of the synthesized salt and diluting to $25 \mathrm{~mL}$ with distilled water. All other chemicals were of analytical reagent grade.

\section{Apparatus}

A 3-axis CNC controlled milling machine, Kit Mill RD300/420 (Originalmind Co., Japan) was used to manufacture $\mu$ SWIA. A portable spectrophotometer, Maya 2000 Pro (Ocean Optics, USA), was used as a detector. Flow controller, Flow controller (PRO-6000) (Yabegawa Co., Japan), comprised of an 11-port multi-way valve and of syringe pump (Yabegawa Co.), was used to build the flow system.

\section{Fabrication texhnique}

The present research and commercialization focus has shifted to microdevices made entirely from polymeric materials. Among polymeric materials prevail polydimethylsiloxane, polycarbonate and polymethylmethacrylate. The main benefits of polymeric microdevices are low cost and durability, which fit field use and point-of-care diagnostic applications. The availability of numerous mechanical and chemical properties achievable by simple chemical changes in the polymer formulations are also important advantages..$^{20-22}$

One of the easiest and cheapest manufacturing techniques of microchips is computer numerical control (CNC) milling, ${ }^{23}$ which is capable of producing microfluidic devices from most solid polymeric materials. CNC milling is used for fabrications PMMA-based microchips, which permits one to make channels with dimensions on the order of $10-500 \mu \mathrm{m}$. CNC milling involves the drilling of channels and holes in a monolithic block and coating with a cover plate. For this technique, well-polished and optically transparent plates should be used. In this work, a PMMA device was fabricated through the direct micro-milling of channels and cavities in a monolithic PMMA plate, followed by bonding it to the cover plate.

Figure 1 presents a scheme of the $\mu$ SWIA. The device was made of two PMMA pieces of $76 \times 52 \times 10 \mathrm{~mm}$. Initially, a draft of the device was designed using the Graphics Editor Cut2D Ver. 1.6 software program. The channels on a PMMA plate were made in accordance with the designed draft by using an automated milling-machine Kit Mill RD300/420. The microflow network of the $\mu$ SWIA contained a mixing chamber

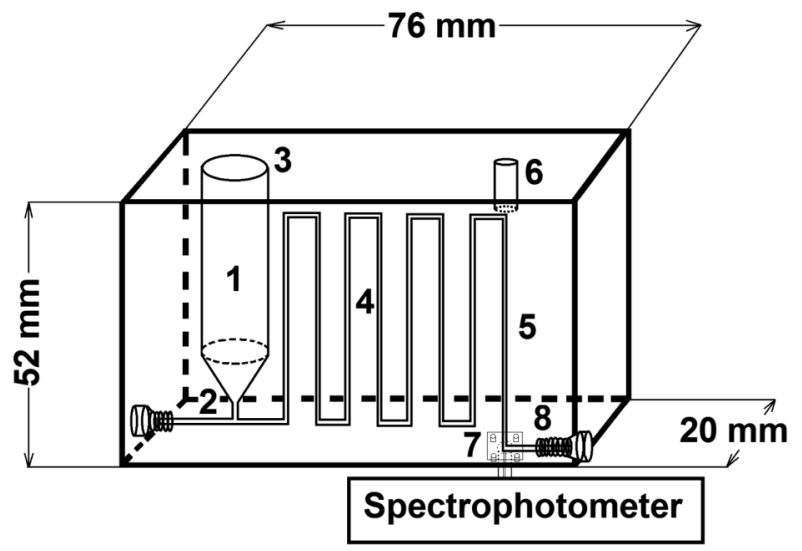

Fig. 1 Scheme of the $\mu$ SWIA. 1. mixing chamber, 2. inlet channel, 3. outlet for air, 4. rectangular channel, 5. optical channel, 6. cavity for LED and ball lens, 7. cavities for optical fiber connection, 8. outlet channel.

(1) a vertically arranged channel $(\phi 5.0 \mathrm{~mm}$, high $26.5 \mathrm{~mm})$ connected by a pyramidal ending with a channel $(2)(\phi 0.5 \mathrm{~mm}$, length $20.5 \mathrm{~mm}$ ) at the bottom and having an outlet (3) at the top. The other important elements of the $\mu$ SWIA were a rectangular labyrinth channel $(4)(\phi 0.5 \mathrm{~mm}$, length $311.4 \mathrm{~mm})$ and an optical channel (5) ( $\phi 0.5 \mathrm{~mm}$, length 36.0).

Two same-size PMMA plates were smoothed and glued together. In the already glued assembly, a hole $(\phi 6.0 \mathrm{~mm}$, depth $18.0 \mathrm{~mm}$ ) for an LED (6) LED 820-01AU (Roithner Laser Technik, Austria) was made on one side of the optical channel (5). The LED used as a light source emitted light at a wavelength $820 \mathrm{~nm}$, exactly corresponding to the absorbance maximum of the reduced 18-MPA. A ball lens of $\phi 5 \mathrm{~mm}, n_{\mathrm{R}}=1.517$ (Edmund Optics, USA, BL-05, uncoated, H-K9L glass) was placed into the channel cavity in front of the LED. On the opposite side of the optical channel (5) a quartz optical fiber of $600 \mu \mathrm{m}$ core diameter (7) QP600-1-SR from Ocean Optics, Inc. was fixed by four screws and used for connection with a spectrophotometer. The surfaces before optical the fiber and inside the cavity for the LED were carefully polished.

\section{Procedure for the $\mu$ SWIA determination of cysteine}

The scheme of the analytical system employed for the $\mu$ SWIA determination of cysteine is shown in Fig. 2. At the first step, the syringe pump was set in the position "Out" for a time interval of $9.00 \mathrm{~s}$ and a sample solution, an acetate buffer solution and a $0.4 \mathrm{mM}$ 18-MPA solution, were sequentially aspirated through the corresponding ports of the multiport valve into the PTFE tube at a rate of $0.2 \mathrm{~mL} \mathrm{~min}^{-1}$ by a backward movement of the syringe pump plunger. The obtained mixture was then transferred by forward movement of the syringe pump from the PTFE tube through channel (1) into the mixing chamber (MC) (2) of the $\mu$ SWIA. After completion of this operation, $100 \mu \mathrm{L}$ of air was injected first into the syringe pump, and then passed through the $\mathrm{MC}$ at a rate of $0.1 \mathrm{~mL} \mathrm{~min}^{-1}$. The multiport valve was changed in the position and the formed heteropoly blue was transferred from the MC through the rectangular channel (3) into the optical channel (4) by the piston syringe pump. The measurement of the absorbance was performed under stopped-flow conditions. At the next step, all components of the manifold were washed with a buffer solution. A measurement of the analytical signal for a blank solution was carried out by the above-mentioned algorithm. However, instead 


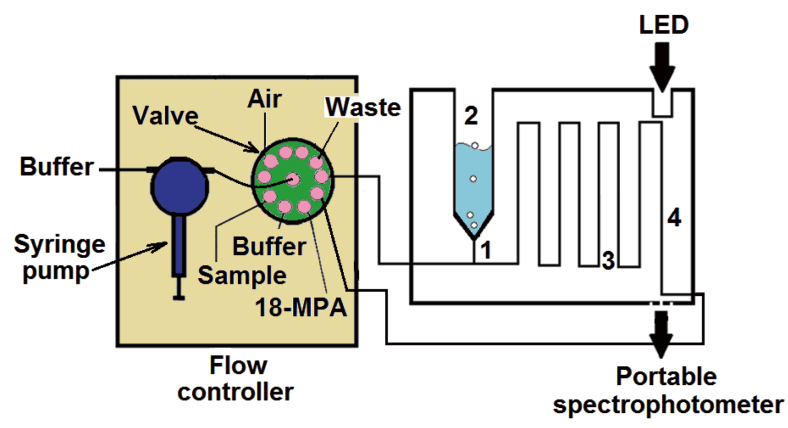

Fig. 2 Scheme of the analytical system for the $\mu$ SWIA determination of cysteine. 1. inlet channel, 2. mixing chamber, 3. rectangular channel, 4. optical channel.

of the sample solution, the buffer solution was aspirated. All of the parameters of the flow analytical system were controlled by software included with the Flow controller PRO-6000. The data acquisition and treatment were performed with software provided along with the Maya Pro 2000 spectrometer.

\section{Sample preparation}

For the analysis of biologically active supplements, the content of one capsule was grounded, $0.35-0.40 \mathrm{~g}$ of the powder was dissolved in distilled water, transferred to a $25 \mathrm{~mL}$ volumetric flask, and the volume was adjusted to the mark with distilled water. The solution was then centrifuged at $5000 \mathrm{rpm}$ for $15 \mathrm{~min}$ and filtered through a $0.45 \mu \mathrm{m}$ membrane filter to separate insoluble components of the biologically active supplements. Before analysis, the sample solution was diluted tenfold, two or three times; an appropriate aliquot of this solution was used for the analysis..$^{24,25}$

\section{Results and Discussion}

\section{Topology of the $\mu$ SWIA}

The topology of the $\mu$ SWIA (Fig. 1) included three main parts: the mixing chamber, the rectangular labyrinth and optical channels. The MC is a vertical cylindrical channel with a pyramidal ending, which was used to connect the MC to the inlet channel. The inlet channel is intended to inject solutions and gas into the MC. The MC design allowed an effective mixing of solutions by gas bubbling.

It was necessary to remove any remaining gas bubbles in the flow before they could reach the optical channel. The specially designed rectangular channel was used to solve this problem. When liquid with bubbles were passing through the horizontal and vertical parts of the rectangular channel at a flow rate of $0.1 \mathrm{~mL} \mathrm{~min}^{-1}$ in turbulent flow, the aqueous and gaseous phases were separated. Thus, the solution reached the optical channel before a gas phase gathered in the channel. The absorbance was measured in the stop-flow mode.

In the previously developed $\mu \mathrm{FBA}$ system, the detection system was integrated into the micro-mixing chamber; the optical path of the $\mu$ FBA photometer was about $6 \mathrm{~mm} .{ }^{19}$ The topology of this device does not allow increasing the optical length path, and consequently the sensitivity. Within the proposed fabrication technique, the optical length path can be varied over a wide range at the stage of the $\mu$ SWIA manufacturing. In accordance with this modification, the path length was increased up to $36.0 \mathrm{~mm}$. To improve the signal/ noise ratio and to exclude the loss of radiation due to scattered light, a ball lens focusing the light emitted from the light source was positioned in the cavity in front of the LED.

Influence of chemical variables on the $\mu$ SWIA determination of cysteine

The reaction between 18-MPA and cysteine is fast and quantitative over a wide range of $\mathrm{pH}$ and reagent concentration. ${ }^{18}$ Optimization of the chemical and flow variables is strongly simplified by using the flow configuration with the MC because most of the optimal values can be directly taken from information obtained by a preliminary investigation of the reaction under batch conditions. The initial set of used parameter values was: $50 \mu \mathrm{L}$ of $0.1 \mathrm{mM}$ cysteine, $50 \mu \mathrm{L}$ of acetate-acetic buffer solution ( $\mathrm{pH} 5.0$ ) and $50 \mu \mathrm{L}$ of 18-MPA solution.

By varying the 18-MPA concentration in the range from 0.1 to $1 \mathrm{mM}$, a constant value of the absorbance was observed, beginning from $0.4 \mathrm{mM}$. Since there was no improvement in the sensitivity for concentrations of 18-MPA higher than $0.4 \mathrm{mM}$, this value was chosen as being optimal.

In order to evaluate the influence of the acidity on the reaction studied, formation of heteropoly blue was studied in the range of medium $\mathrm{pH}$ from 2.0 to 10.0. Hydrochloric acid (from $3.0 \mu \mathrm{M}$ to $30 \mathrm{mM}$ ) or a borate buffer solution ( $\mathrm{pH}$ from 7.8 to 10) was used for $\mathrm{pH}$ adjustment. The reaction was too slow at a $\mathrm{pH}$ of less than 5.0. At a higher $\mathrm{pH}$, the rate of the reaction is sufficiently rapid to be appreciable, but at a $\mathrm{pH}$ of more than 6.0 the hydrolysis of 18-MPA is greatly increased. $\mathrm{A} \mathrm{pH}$ of 5.0 adjusted with an acetate-acetic buffer solution ( $\mathrm{pH}$ 5.0) was accepted as being to optimal acidity value.

The total volume of the reaction mixture was minimized so as to provide the minimum consumption of the sample and the reagent, waste generation, and the proper reproducibility $(\mathrm{S}<$ $1.7 \%$ ). For $10 \mu \mathrm{L}$ of $0.1 \mathrm{mM}$ cysteine, increasing the volume of the 18-MPA increased the signal intensity, which reached a plateau at a volume of $10 \mu \mathrm{L}$. That value was selected as being optimal; $10 \mu \mathrm{L}$ of the buffer solution was sufficient to ensure $\mathrm{pH} 5.0$ of the reaction mixture.

The effect of time while the reaction components were being mixed with air in the MC on the efficiency of solution homogenization and completeness of the chemical reaction was investigated in the air flow-rate range from 0.05 to $0.5 \mathrm{~mL} \mathrm{~min}^{-1}$. An air flow rate of $0.1 \mathrm{~mL} \mathrm{~min}^{-1}$ was chosen as being optimal because at higher values a carryover of the solution from the $\mathrm{MC}$ can occur.

\section{Analytical performance}

An overview of the optimal conditions for the MSWIA determination of cysteine is given in Table 1. A calibration graph was linear in the range of cysteine concentrations from 0.009 to $0.1 \mathrm{mM}$. The limit of detection, determined as 3-times the standard deviation of 10 replicate blank measurements, was equal to $3 \mu \mathrm{M}$. The precision was examined using the RSD values: for two concentration levels $(0.009$ and $0.1 \mathrm{mM})$, the RSD values constitute 1.5 and $1.7 \%$, respectively. The $\mu$ SWIA procedure was characterized by reasonable for most applications throughput of up to 12 samples $\mathrm{h}^{-1}$.

\section{Interference study}

The influence of those compounds possessing reducing properties, including molecules having phenolic and hydroxylic groups, as well as some other substances or excipients on the determination of cysteine, was evaluated. Increasing concentrations of possible interfering agents were added to a solution mixture with a fixed amount $(0.05 \mathrm{mM})$ of cysteine, 
Table 1 Characteristics of the proposed method for the $\mu$ SWIA determination of cysteine

\begin{tabular}{lc}
\hline \multicolumn{1}{c}{ Parameter } & Found or optimal value \\
\hline $\mathrm{pH}$ & 5.0 \\
18-MPA concentration/mM & 0.4 \\
Volume of sample/ $\mu \mathrm{L}$ & 10 \\
Volume of $18-\mathrm{MPA} / \mu \mathrm{L}$ & 10 \\
Time of mixing in $\mathrm{MC} / \mathrm{s}$ & 60 \\
Linear range/mM & $0.009-0.1$ \\
Calibration graph & \\
Slope & 6200 \\
Intercept & 0.013 \\
Correlation coefficient $\left(R^{2}\right)$ & 0.997 \\
Limit of detection/ $\mu \mathrm{M}$ & 3 \\
Sampling rate $($ per hour $)$ & 12 \\
RSD, $\%(n=10)$ & 1.7 \\
\hline
\end{tabular}

Table 2 Influence of some interfering species on the determination of $0.05 \mathrm{mM}$ of cysteine

\begin{tabular}{lc}
\hline \multicolumn{1}{c}{ Species } & $\begin{array}{c}\text { Tolerance } \\
\text { concentration/mM }\end{array}$ \\
\hline Sucrose, paracetamol & $10^{\mathrm{a}}$ \\
Uric acid & 0.1 \\
Citric acid, tartaric & $10^{\mathrm{a}}$ \\
Salicylic acid, acetylsalicylic acid & 0.5 \\
Norepinephrine, epinephrine, methyldopa & 0.4 \\
Ascorbic acid & 0.01 \\
Glutathione & 0.01 \\
Lysine, serine, glycine, alanine, valine, methionine & $0.5^{\mathrm{a}}$ \\
\hline
\end{tabular}

a. Highest concentration studied.

and the corresponding analytical signals were recorded. The tolerance concentration was defined as the maximum concentration of some potential interfering substance that would cause a change in the signal of $\pm 5 \%$. The results are given in Table 2. The interferences from glutathione and ascorbic acid were significant, beginning from $0.01 \mathrm{mM}$. Oxoacids in of concentrations of up to $10 \mathrm{mM}$ had no influence. None of the amino acids studied interfered with the determination of cysteine by the proposed method.

\section{Analytical application}

The developed method was applied to the determination of cysteine in biologically active food supplements and fodder (Table 3). The content of cysteine found by the proposed procedure in tablets was close to the labeled value. A highperformance liquid chromatography method (HPLC) was used to confirm the accuracy of the obtained results. ${ }^{24}$ Paired $t$-tests show that the cysteine contents found by the $\mu$ SWIA method were insignificantly different from those obtained by HPLC method at a $95 \%$ confidence level.

\section{Conclusions}

In this paper, the development of a new micro-stepwise injection spectrophotometric analyzer has been described. The $\mu$ SWIA manufacturing was performed by the $\mathrm{CNC}$ milling technique, which allowed us to fabricate the channels in devices of different size and arrangement. A new $\mu$ SWIA system provides rapid
Table $3 \mu$ SWIA and HPLC determination of cysteine in biologically active supplements and fodder ( $\mathrm{mg}$ of cysteine/ capsule \pm confidence intervals of the method for $n=5$ and $95 \%$ confidence level)

\begin{tabular}{lcccc}
\hline \multicolumn{1}{c}{ Sample } & $\begin{array}{c}\text { Labeled } \\
\text { value }\end{array}$ & $\begin{array}{c}\mu \text { SWIA } \\
\text { manifold }\end{array}$ & $\begin{array}{c}\text { HPLC } \\
\text { method }\end{array}$ & $t$-test \\
\hline Fitoval (KRKA) & $100 \mathrm{mg}$ & $110 \pm 13 \mathrm{mg}$ & $110 \pm 18 \mathrm{mg}$ & 0.64 \\
Revalid (TEVA) & $50 \mathrm{mg}$ & $53 \pm 4 \mathrm{mg}$ & $54 \pm 9 \mathrm{mg}$ & 0.83 \\
Fodder & $1 \mathrm{~g} \mathrm{~kg}^{-1}$ & $1.22 \pm 0.08 \mathrm{~g} \mathrm{~kg}^{-1}$ & $1.17 \pm 0.09 \mathrm{~g} \mathrm{~kg}^{-1}$ & 1.70 \\
\hline
\end{tabular}

and effective homogenization of the reaction mixture components and completion of the reaction by means of gas bubbling. The interfering effect of random appearing bubbles on the absorbance measurement was effectively eliminated by the proposed channel topology. The use of air for reactants homogenization in the MC simplifies the configuration of the flow system, making it more autonomous, light and cheap. An important advantage of the $\mu$ SWIA is the simplicity of manufacturing, and reduced cost of the raw materials (PMMA). It is possible to combine the $\mu$ SWIA with various types of detectors.

The undoubted advantage of $\mu$ SWIA over the SWIA manifold is the conjunction of homogenization and detection systems on the same device. This option was provided so as to change the volume of the mixing chamber in order to minimize the consumption of regents, and also allowed us to vary the optical pass length, which is important for spectrophotometric detection. It should be pointed out that in the case of $\mu$ SWIA the production of waste and the consumption of the sample and reagent solutions are lower than that in the previously proposed SWIA method for cysteine determination in biologically active supplements and fodders (60-times less). ${ }^{18}$ However, the throughput of $\mu$ SWIA is less than that in SWIA (almost twice) due to the different types of pumps being used (syringe and peristaltic pumps, respectively).

\section{Acknowledgements}

We gratefully acknowledge financial support of the Russian Foundation for Basic Research (Grant No. 13-03-00031). This work was supported by the Ministry of Education and Science of the Russian Federation in form of Scholarship of the Russian President (Order No. 539). Scientific research was performed at the Center for chemical analysis and materials research of St. Petersburg State University.

\section{References}

1. D. J. Harrison, K. Fluri, K. Seiler, Z. Fan, C. S. Effenhauser, and A. Manz, Science, 1993, 261, 895.

2. A. Manz, N. Graber, and H. M. Widmer, Sens. Actuators, B, 1990, $1,244$.

3. A. Marx, J. C. Fettinger and E. Verpoorte, H. Liidi, H. M. Widmer, and D. J. Harrison, TrAC, Trends Anal. Chem., 1991, 10, 84 .

4. H. Liidi, M. B. Garn, S. D. Haemmerli, A. Manz, and H. M. Widmer, J. Biotechnol., 1992, 25, 75.

5. A. Gałuszka, Z. Migaszewski, and J. Namieśnik, TrAC, Trends Anal. Chem., 2013, 50, 78.

6. S. Xue, K. Uchiyama, and H.-F. Li, J. Environ. Sci., 2012, 
$24,564$.

7. J. Ruzicka, Analyst, 2000, 125, 1053.

8. M. Miro and E. H. Hansen, Anal. Chim. Acta, 2007, 600, 46.

9. P. Phansi, C. Henríquez, E. Palacio, D. Nacapricha, and V. Cerdá, Talanta, 2014, 119, 68.

10. F. Z. Abouhiat, C. Henríquez, B. Horstkotte, F. El Yousfi, and V. Cerdá, Talanta, 2013, 108, 92.

11. S. Guo and T. Imato, J. Flow Injection Anal., 2013, 30, 29.

12. S. Guo and T. Imato, J. Flow Injection Anal., 2013, 30, 21.

13. P. H. G. D. Diniz, L. F. de Almeida, D. P. Harding, and M. C. U. de Araujo, TrAC, Trends Anal. Chem., 2012, 35, 39.

14. G. Marshall, D. Wolcott, and D. Olson, Anal. Chim. Acta, 2003, 499, 29.

15. A. V. Bulatov, A. V. Petrova, A. B. Vishnikin, A. L. Moskvin, and L. N. Moskvin, Talanta, 2012, 96, 62.

16. A. V. Bulatov, I. I. Timofeeva, and A. L. Moskvin, J. Flow Injection Anal., 2013, 30, 51.

17. A. Bulatov, M. Soloviev, A. Petrova, A. Moskvin, and L. Moskvin, J. Flow Injection Anal., 2010, 27, 158.
18. A. V. Bulatov, A. V. Petrova, A. B. Vishnikin, and L. N. Moskvin, Microchem. J., 2013, 110, 368.

19. S. S. Monte-Filho, M. B. Lima, S. I. E. Andrade, D. P. Harding, Y. N. M. Fagundes, S. R. B. Santos, S. G. Lemos, and M. C. U. Araújo, Talanta, 2011, 86, 208.

20. H. Becker and L. E. Locassio, Talanta, 2002, 56, 267.

21. D. F. Pozo-Ayuso, M. Castano-Alvarez, A. Fernandez-laVilla, M. García-Granda, M. T. Fernández-Abedul, A. Costa-García, and J. Rodríguez-García, J. Chromatogr. A, 2008, 1180, 193.

22. H. Zhai, J. Li, Z. Chen, Z. Su, Z. Liu, and X. Yu, Microchem. J., 2014, 114, 223.

23. J. S. Mecomber, D. Hurd, and P. A. Limbach, Int. J. Mach. Tools Manu., 2005, 45, 1542.

24. A. O. Rudenko, L. A. Karcova, and S. I. Snarski, Sorption Chromatogr. Processes, 2012, 10, 233.

25. State Standard of the Russian Federation No. 13496.22-90. Fodders, mixed fodders and mixed fodder raw materials. Method for the determination of cysteine and methionine. 